\title{
Indicadores educacionais no Ensino Superior Brasileiro: possíveis articulações entre desempenho e características do alunado
}

\author{
Anandra Santos Ribeiro de Oliveira
}

Ivair Ramos Silva

\begin{abstract}
Resumo: A discussão sobre aproveitamento escolar e sua relação com a origem social é investigada pela sociologia da educação desde a segunda metade do século XX. No Brasil, em específico, vem se produzindo um grande volume de pesquisas sobre essa temática no nível da educação básica, a partir de análises que discutem desempenho alcançado, verificado por meio de resultados de avaliações educacionais de larga escala, e características socioeconômicas dos alunos. No entanto, sob essa perspectiva, pouco tem se pesquisado no ensino superior. Nesta linha, este artigo analisa a relação entre o aproveitamento acadêmico de alunos de graduação brasileiros e seu contexto socioeconômico. Foi feito estudo empírico baseado nos dados do Exame Nacional de Desempenho dos Estudantes (ENADE), obtendo-se evidências sobre as características que influenciam no desempenho dos alunos, tais como etnia, renda e tipo de instituição de ensino. Vale destacar o efeito compensador que parece existir entre a elevada escolaridade dos pais e a baixa renda familiar.
\end{abstract}

Palavras-chave: Ensino superior. Estratificação social. Desempenho acadêmico.

\section{Education Indicators in Higher Education Brazilian: possible interactions between performance and characteristics of the students}

Abstract: The relationship between social group position and educational performance has been debated by sociologists since the second half of the twentieth century. In Brazil, in particular, a large volume of research on this subject focused on the basic education level has been produced, from analyses about performance achieved, verified by results of large-scale educational assessments, and socioeconomic characteristics of the students. However, from this perspective, little has been studied in higher education. In this paper, the objective is to analyze such relationship among undergraduate Brazilian students. Through statistical analyses, based on the "Exame Nacional de Desempenho dos Estudantes (ENADE)", we have obtained consistent evidences about the correspondence between the social characteristics of students, such as race and family income, and their performance in the college.

Key words: Higher Education. Social stratification. Academic performance.

Este é um artigo publicado em acesso aberto sob uma licença Creative Commons

https://creativecommons.org/licenses/by-nc/4.0/ 


\section{Introdução}

A partir dos anos 1960, inicia-se na Sociologia da educação a problematização da origem das desigualdades escolares, face ao descrédito diante do propalado paradigma funcionalista de que as instituições educacionais possibilitariam aos indivíduos obter, igualitariamente, retorno social e econômico, materializados pela mobilidade ascendente na estrutura de classes. No entanto, frustradas essas expectativas, frente à desvalorização dos certificados escolares e ao inexplicado insucesso escolar de muitos, instaura-se, na década de 60, uma profunda crise sobre a concepção do papel da escola na sociedade ocidental. Nogueira e Nogueira (2002) apontam esse período como um marco para a reinterpretação radical do papel social dos sistemas de ensino, trazendo como aspecto positivo o reconhecimento da origem social dos alunos como fator a ser considerado para explicar o desempenho e trajetória escolar, até então desconsiderada em razão da lógica da meritocracia. Essa que, apregoada pela escola funcionalista, conduziria ao pensamento de que o aproveitamento escolar poderia ser explicado, única e exclusivamente, por dons individuais. Este artigo pretende, assim, inserir-se nessa discussão ao analisar a relação entre o aproveitamento acadêmico de alunos de graduação, por meio na nota obtida no ENADE, e seu contexto socioeconômico, descrito pelo perfil dos alunos e características da Instituição de Ensino Superior. Para isso foi feito estudo empírico, utilizando-se os dados do Exame Nacional de Desempenho dos Estudantes (ENADE), por meio de modelos de regressão linear múltipla. Justifica-se a temática como de extrema relevância por haver poucos estudos que tratem do ensino superior, sob essa perspectiva. As demais pesquisas, em sua maioria, referem-se à Educação Básica (ALVES; SOARES, 2001; SOARES; MAROTTA, 2009; LAROS; MARCIANO; ANDRADE, 2010). Além do mais, pode-se, com esse estudo, contribuir para o importante paradigma utilizado na interpretação sociológica da educação, que considera a origem social como condição primordial para se pensar a trajetória escolar.

Organizado em quatro seções, além da introdução, o texto a seguir trata, na primeira parte, de uma breve revisão sobre estratificação social e aproveitamento escolar. Em seguida, explica-se a abordagem analítica, em que será detalhado o modelo estatístico utilizado e sua operacionalização, a partir da contextualização teórica das variáveis escolhidas. Serão apresentados, ainda, os resultados e a discussão em torno dos mesmos. 
Indicadores educacionais no Ensino Superior Brasileiro: possíveis articulações entre desempenho e características do alunado

\section{Refletindo sobre estratificação social e aproveitamento escolar}

A reinterpretação radical do papel da escola na trajetória escolar contribuiu, entre outras coisas, para a descoberta da forte interação entre aproveitamento escolar e origem social, em detrimento da perspectiva que apenas considerava as características individuais (biológicas e psicológicas) como fatores associados ao desempenho escolar. Essa nova leitura, iniciada na década de 1960, teve como substrato teórico a discordância entre duas correntes, a Estruturalfuncionalista (1945-1965), na figura de Talcott Parsons, e a Teoria da Reprodução (1965-1975), representada por Pierre Bourdieu (2001). Enquanto a primeira tratava a escola como neutra em suas ações educativas e, portanto, promotora de equidade social, a segunda, pressupõe a escola não mais como desconectada da estrutura de classes. Ao contrário, as instituições educacionais serviriam como mecanismo de reprodução e legitimação das desigualdades sociais, ao mesmo tempo em que as dissimularia sob a lógica aparente da meritocracia (CUNHA, 2010, p. 41). Percebe-se, assim, o enfraquecimento da lógica do mérito, proposta pelo paradigma funcionalista, dando lugar aos pressupostos da Teoria da Reprodução, que vinculam a trajetória escolar à origem social. Associa-se, ainda, o enfraquecimento do paradigma funcionalista à divulgação de uma série de extensas pesquisas quantitativas patrocinadas pelos governos inglês, americano e francês (Aritmética Política inglesa, Relatório Coleman - EUA, Estudos do INED, França), na medida em que ressaltaram de forma sistemática, o peso da origem social sobre a trajetória escolar, em detrimento dos dons individuais. Soma-se a isso, o fato de que os resultados iniciais dessas pesquisas, creditados a deficiências passageiras do sistema educacional, não se mostravam melhores, mesmo após maciços investimentos governamentais. (NOGUEIRA; NOGUEIRA, 2002, p. 17).

Frente a esse novo cenário, a Teoria da Reprodução, representada por Pierre Bourdieu e também inserida no conjunto das Teorias Crítico-Reprodutivistas, contribuiu para que a origem social passasse a ser concebida como condição para gerar situações mais ou menos favoráveis face às exigências escolares. Passa-se, assim, a considerar a bagagem socialmente herdada como elemento central de análise, na medida em que ela pode ser colocada a serviço do sucesso ou insucesso acadêmico. Apesar das críticas feitas ao trabalho de Bourdieu, de supervalorizar a visão pessimista sobre a escola e da leitura de outros autores afirmando que nem sempre as desigualdades sociais se reproduzem completamente na sala de aula, seu enquadramento teórico não foi superado e vêm inspirando pesquisadores em todo o mundo, desde a década de 1970. Haja vista os estudos desenvolvidos na área educacional por autores 
como Bourdieu (2001); Nogueira e Nogueira (2002, 2009); Nogueira (2004); Bonamino et al. (2010); Oliveira e Melo-Silva (2010).

\section{Abordagem analítica}

Os resultados que serão apresentados a seguir foram obtidos a partir de estudo quantitativo, que utilizou como recurso metodológico para tratamento dos dados o modelo de regressão linear. A escolha por essa abordagem analítica justifica-se pelo objetivo proposto de verificar se há associação entre a variável resposta - aproveitamento acadêmico interpretado por meio das notas obtidas na prova do ENADE - e o contexto sócio econômico do aluno, representado pelas variáveis explicativas, raça/cor; sexo; renda familiar, situação de trabalho, escolaridade dos pais, que tipo de escola cursou o ensino médio e categoria administrativa da Instituição de Ensino Superior a qual estava vinculado no momento da coleta de dados. Elas serão melhor descritas no tópico seguinte. Os dados utilizados no modelo foram extraídos dos microdados do ENADE, disponibilizados pelo Instituto Nacional de Estudos e Pesquisas Educacionais Anísio Teixeira (INEP), do ano de 2012, totalizando a participação de 480.687 alunos do ensino superior de todo o Brasil. Foram incluídos na amostra os alunos presentes, ingressantes e concluintes dos cursos avaliados no ano de 2012 pelo ENADE, que foram os seguintes: Administração, Ciências Contábeis, Ciências Econômicas, Design, Direito, Jornalismo, Psicologia, Publicidade e Propaganda, Relações Internacionais, Secretariado Executivo e Turismo. Dos cursos de graduação tecnológica estão inclusos Tecnologia em Gestão Comercial, em Gestão de Recursos Humanos, em Gestão Financeira, em Logística, em Marketing e em Processos Gerenciais.

\subsection{Explicação das variáveis inseridas no modelo conceitual}

Esta seção traz uma discussão sobre o que motivou a escolha das variáveis utilizadas no modelo conceitual em que este trabalho se baseia. Na oportunidade, inseriu-se, também, a transcrição das questões da forma como foram apresentadas aos respondentes.

\section{- Aproveitamento acadêmico interpretado por meio das notas obtidas na prova do ENADE}

A discussão e a preocupação sobre a qualidade do ensino no Brasil fomentaram, a partir da década de 1990, a implantação da avaliação educacional nos diferentes níveis de ensino em moldes sistematizados. No ensino superior brasileiro, em específico, as avaliações de larga 
Indicadores educacionais no Ensino Superior Brasileiro: possíveis articulações entre desempenho e características do alunado

escala para esse nível iniciaram-se em 1996, com a criação do Exame Nacional de Cursos (ENC), também conhecido como Provão. Esse instrumento foi elaborado pelo INEP, com o propósito de avaliar a qualidade dos cursos de graduação, por meio da verificação do desempenho de seu respectivo alunado. Extinto em 2003, ele foi substituído, em 2004, pelo ENADE. O ENADE operacionaliza-se por meio de quatro instrumentos, a saber: as provas de conhecimento geral e específico; um questionário para levantar a percepção dos alunos sobre o teste, que faz parte do caderno de prova; um questionário sobre o perfil sócio-econômicoeducacional do aluno e um questionário a ser respondido pelo coordenador de curso sob avaliação, no qual se pede suas impressões sobre o projeto pedagógico e as condições gerais de ensino de seu curso. Nesse artigo, utilizou-se, para a construção da análise dos dados, as informações geradas pelo questionário sócio-econômico-educacional e a nota da prova de conhecimento geral e específico.

\section{- Raça/cor/etnia (Etnia) - Questão: Como você se considera?}

$\mathrm{O}$ acesso ao ensino superior brasileiro é caracterizado por um maior acesso de pessoas declaradas brancas, seguido de pardas e negras. Em uma última divulgação do Instituto Brasileiro de Geografia e Estatística (IBGE), do ano de 2009, encontraram-se os valores da participação de alunos brancos $(62,6 \%)$ e pretos e pardos $(37,4 \%)$. Para além dessa estratificação de acesso, já conhecido, vários estudos têm sido realizados sobre o desempenho de alunos considerando-se a raça/cor/etnia como ponto de análise. Foram encontradas evidências que apontam alunos brancos como tendo desempenho superior em comparação aos alunos que se declaram pardos, pretos, amarelos ou indígenas. Soares e Marotta (2009); Barros et al., (2001); Henriques (2001); Andrade e Dachs (2007); Alves e Soares, (2001). Por esse motivo, torna-se relevante averiguar se esse padrão de desempenho também se verifica nessa amostra estudada.

- Renda familiar - Questão: Somando a sua renda com a renda dos familiares que moram com você, quanto é, aproximadamente, a renda familiar? (Considere a renda de todos os seus familiares que moram na sua casa com você)

Compreende-se, a partir da perspectiva de Pierre Bourdieu (2001), que o indivíduo é caracterizado, também, por uma bagagem socialmente herdada, que inclui, dentre outros capitais, o capital econômico. Esse que, traduzido em linhas gerais, pode ser compreendido como responsável pelo acesso a bens e serviços, quais sejam, frequentar excelentes instituições de ensino, bens culturais, viagens de estudo, melhor infraestrutura para estudo em casa, entre 
outros. Esses acessos, quando vivenciados, poderiam impactar positivamente a trajetória escolar de alunos, na medida em que aproximam o estudante dos princípios da classe dominante praticados nas instituições de ensino, a partir de suas práticas pedagógicas. Partindo-se desse entendimento, encontram-se evidências de que alunos provenientes de famílias com maior renda, isto é, com maior capital econômico, costumam apresentar melhores resultados escolares. Soares, Martins e Assunção (1998); Bonamino et al. (2010); Alves (2012).

\section{- Situação de trabalho - Questão: Indique a resposta que melhor descreve sua atual situação de trabalho. (Não contar estágio, bolsas de pesquisa ou monitoria)}

Em uma leitura corrente, os alunos que trabalham e estudam, simultaneamente, poderiam assim fazê-lo por estarem em condições socioeconômicas desfavoráveis. E o fato de estudar poderia levar a piores resultados, em razão de uma menor disponibilidade para se dedicar aos estudos. No entanto, Cardoso e Sampaio (1994) problematizam a situação afirmando não ser possível restringir a explicação do trabalho do estudante a condicionantes socioeconômicos unicamente. Embora sejam importantes para justificar boa parte dos casos de estudantes que trabalham, eles não dão conta de todas as variáveis que envolvem a opção pelo emprego. Em consonância com o argumento das autoras acima, Nogueira (2004) encontrou em recente pesquisa com famílias com alto poder aquisitivo, jovens que conciliavam estudo e trabalho. Para esses jovens, o trabalho não se devia ao auto-sustento ou à complementação do orçamento familiar, mas por uma necessidade de conhecer o mundo profissional. Por outro lado, Lordêlo (2004) encontrou evidências de que a frequência de estudantes que trabalham e estudam vai diminuindo nas faixas de renda mais altas, fazendo-se uma associação entre necessidade de trabalhar e renda. Diante dessas várias leituras sobre a relação entre trabalho e estudo, essa categoria foi incluída no modelo conceitual para identificação de como os resultados encontrados se apresentam diante dessa tríade desempenho, situação ocupacional e posição socioeconômica.

\section{- Escolaridade dos pais - Questões: Até que nível de ensino seu pai estudou? Até que nível de ensino sua mãe estudou?}

Considerou-se relevante avaliar a escolaridade dos pais, tendo em vista o conceito de capital cultural proposto por Bourdieu (2001). A propósito, como nos mostra Alves (2010), o grau de escolaridade dos pais é o indicador mais utilizado para medir o capital cultural das famílias, porque constitui a forma institucionalizada desse capital, na forma de títulos reconhecidos. Em estudo realizado pela mesma autora, para composição do nível 
Indicadores educacionais no Ensino Superior Brasileiro: possíveis articulações entre desempenho e características do alunado

socioeconômico de alunos, foram encontradas evidências de que filhos de pai com escolaridade média ou superior têm desempenho escolar melhor do que aqueles cujos pais têm menor escolaridade. Encontram-se, ainda, evidências do impacto do nível de escolaridade dos pais na vida dos filhos em trabalhos como o de Oliveira e Melo-Silva (2010), Nogueira e Nogueira (2002), entre outros.

\section{- Tipo de escola em que cursou o ensino médio - Questão: Em que tipo de escola você cursou o ensino médio?}

Historicamente, como aponta Ortega (2001) faz-se uma distinção entre a qualidade do ensino médio praticado em escolas públicas e privadas, de forma que isso afetaria o desempenho em avaliações, bem como o acesso a etapas de escolaridade seguintes, tal qual a entrada no ensino superior. Em específico, o ensino médio particular tem se destacado nas avaliações realizadas, como no Exame Nacional do Ensino Médio (ENEM) e, inclusive, no vestibular. Castro e Tiezzi (2005); Moraes e Nunes (2010). Tais diferenças poderiam ser atribuídas a infraestrutura, corpo docente, perfil socioeconômico do alunado, projeto pedagógico institucional, entre outros fatores. Partindo-se, portanto, do pressuposto da diferença de qualidade entre as redes de ensino e de que ela seria melhor nas escolas particulares, de modo que os alunos da rede privada estariam mais bem preparados para os desafios escolares, torna-se relevante considerar essa variável para avaliar se o tipo de escola em que se cursou o ensino médio, pública ou privada, teria alguma associação com o desempenho no ENADE.

\section{- Categoria administrativa da Instituição de Ensino Superior}

$\mathrm{Na}$ Educação Superior, é frequente a leitura de que as instituições públicas são consideradas de melhor qualidade e que atraem melhores candidatos. Oliveira e Melo-Silva (2010), afirmam que isso pode ser observado por meio dos exames de ingresso às IES públicas, na medida em que sendo a relação candidato/vaga grande, são selecionados os candidatos melhor preparados para o tipo de prova que é realizada. A título de exemplificação, tem-se o último censo da educação superior divulgado que traz a relação candidato vaga de $7,1 \mathrm{em}$ instituições públicas, contra 1,2 em instituições privadas (INEP, 2008). Assim, poder-se-ia supor que as IES públicas teriam maior qualidade em relação às instituições privadas, tendo em vista que a primeira conteria os melhores alunos. Dentre as evidências encontradas sobre essa hipótese, há o estudo de Soares, Martins e Assunção (1998), sobre candidatos ao vestibular de uma instituição pública e outra privada. Os autores encontraram evidências de que os resultados 
médios dos alunos matriculados na Instituição pública são maiores que dos alunos matriculados na Instituição Privada. Resta saber se essa diferença de desempenho inicial também se verifica nos demais resultados auferidos ao longo dos anos de graduação. Assim, torna-se relevante considerar essa variável para avaliar se a categoria administrativa, pública ou privada, tem associação com a condição socioeconômica e desempenho observado.

\section{Análises estatísticas}

Após as justificativas teóricas descritas na última seção, apresenta-se a seguir o modelo linear conceitual para a representação matemática da relação entre as variáveis adotadas e a nota no ENADE. A nota geral no ENADE do respondente i, expressa como função das variáveis adotadas, tem a seguinte forma:

$$
\operatorname{ENADE}_{i}=g\left(X_{i}\right)+e_{i}
$$

onde $e_{i}$ é um ruído aleatório tal que $\operatorname{Var}\left(\mathrm{e}_{\mathrm{i}}\right)=\sigma_{\mathrm{e}}^{2}, \mathrm{E}\left(\mathrm{e}_{\mathrm{i}}\right)=0$ para todo $\mathrm{i}=1, \ldots, \mathrm{n}$, em que $\mathrm{n}$ é o número total de respondentes, e $\mathrm{E}\left(\mathrm{e}_{\mathrm{i}} \mathrm{e}_{\mathrm{k}}\right)=0$ para todo $\mathrm{i} \neq \mathrm{k}$. Veja que a usual suposição de normalidade para a distribuição de $\mathrm{e}_{\mathrm{i}}$ é dispensada aqui, o que é possível por termos um elevado número de observações $(n=480.687)$, garantindo assim uma boa aproximação pelas distribuições assintóticas dos estimadores dos coeficientes do modelo. A função de valor real $\mathrm{g}\left(\mathrm{X}_{\mathrm{i}}\right)$ é uma combinação linear das covariáveis do vetor $\mathrm{X}_{\mathrm{i}}$, cujas entradas são formadas pelas seguintes variáveis: etnia, renda familiar, situação de trabalho, escolaridade dos pais, tipo de ensino médio, e categoria da IES. Como neste trabalho todas as covariáveis são de natureza qualitativa, tal combinação linear é possível pela representação de suas categorias por variáveis indicadoras (dummies). Após discutirem-se as relações teóricas de cada covariável com a nota no ENADE, também suportadas pelas análises descritivas da próxima seção, uma representação mais clara para o modelo (1) será fornecida na Seção 4.2.

\subsection{Descrição e exploração dos dados}

As análises descritas a seguir buscam verificar se as relações teóricas, estabelecidas no modelo conceitual, entre covariáveis e desempenho dos alunos no ENADE, são coerentes com o que se obtém empiricamente. Como já mencionado, o ano de 2012 totalizou a participação de 587.351 alunos, porém, existem informações faltantes para uma ou mais variáveis nos questionários de alguns alunos, o que inviabiliza sua utilização na modelagem estatística aqui proposta. Com isto, o número total efetivo de observações utilizadas nesta análise foi de 
Indicadores educacionais no Ensino Superior Brasileiro: possíveis articulações entre desempenho e características do alunado

480.687 indivíduos. Para estes, a média global do ENADE foi de aproximadamente 38,1. Os valores mínimo e máximo foram 0 e 94, respectivamente, enquanto que o primeiro, segundo e terceiro quartis foram, nesta ordem, iguais a 28,1,37,7 e 47,7. Apenas 11,36\% dos respondentes são de instituições de ensino superior públicas, os quais apresentaram um ENADE médio de 41,16, contra a média de 37,71 observada entre os alunos de IES privadas. Apresenta-se a seguir as frequências observadas para cada possível resposta em cada variável de interesse.

Tabela 1 - Frequências relativas por categorias das variáveis Etnia e Tipo de escola onde cursou o ensino médio

\begin{tabular}{ccccccc|c}
\hline & \multicolumn{7}{c}{ Tipo de Escola que cursou no ensino médio } \\
& \multicolumn{1}{c}{ A } & B & C & D & E & Global \\
\cline { 2 - 7 } & A & $35,87 \%$ & $20,00 \%$ & $3,70 \%$ & $2,85 \%$ & $1,76 \%$ & $64,18 \%$ \\
& B & $4,90 \%$ & $1,01 \%$ & $0,37 \%$ & $0,21 \%$ & $0,17 \%$ & $6,66 \%$ \\
& C & $17,95 \%$ & $5,63 \%$ & $1,52 \%$ & $1,01 \%$ & $0,71 \%$ & $26,82 \%$ \\
& D & $0,90 \%$ & $0,58 \%$ & $0,11 \%$ & $0,09 \%$ & $0,05 \%$ & $1,73 \%$ \\
& E & $0,36 \%$ & $0,15 \%$ & $0,04 \%$ & $0,03 \%$ & $0,02 \%$ & $0,60 \%$ \\
\hline & Global & $59,98 \%$ & $27,37 \%$ & $5,74 \%$ & $4,19 \%$ & $2,71 \%$ & $100,00 \%$ \\
\hline
\end{tabular}

Fonte: Instituto Nacional de Estudos e Pesquisas Educacionais Anísio Teixeira

A Tabela 1 contém a distribuição conjunta das frequências relativas dos respondentes das variáveis Etnia e Tipo de escola onde cursou o segundo grau. As categorias para a variável Etnia são: $\mathrm{A}=\operatorname{Branco}(\mathrm{a}) ; \mathrm{B}=\operatorname{Negro}(\mathrm{a}) ; \mathrm{C}=\operatorname{Pardo}(\mathrm{a}) /$ mulato(a); $\mathrm{D}=\operatorname{Amarelo(a)}($ de origem oriental); E = Indígena ou de origem indígena. Para a variável Tipo de escola, temos: A = Todo em escola pública; $\mathrm{B}=$ Todo em escola privada (particular); $\mathrm{C}=\mathrm{A}$ maior parte em escola pública; $\mathrm{D}=\mathrm{A}$ maior parte em escola privada (particular), e $; \mathrm{E}=$ Metade em escola pública e metade em escola privada (particular). Observa-se, dentre os valores encontrados, que cerca de $60 \%$ dos respondentes cursaram todo o ensino médio em escola pública, e que em torno de $64 \%$ são de etnia branca. Para avaliar descritivamente o desempenho no ENADE dos alunos estratificados conforme estas variáveis, apresentam-se os gráficos da Figura 1. Para que se tenha uma referência sobre os desempenhos dos subgrupos de alunos, a linha horizontal pontilhada, presente em ambos os gráficos, indica a média global $(38,1)$ obtida no ENADE. Pode-se observar que os respondentes de etnia branca e os que cursaram todo o ensino médio em escola privada, apresentam as maiores medianas no ENADE, sendo que estes últimos apresentaram uma mediana acima da média global. 
Figura 1 - ENADE 2012 por etnia e tipo de escola durante o ensino médio do respondente
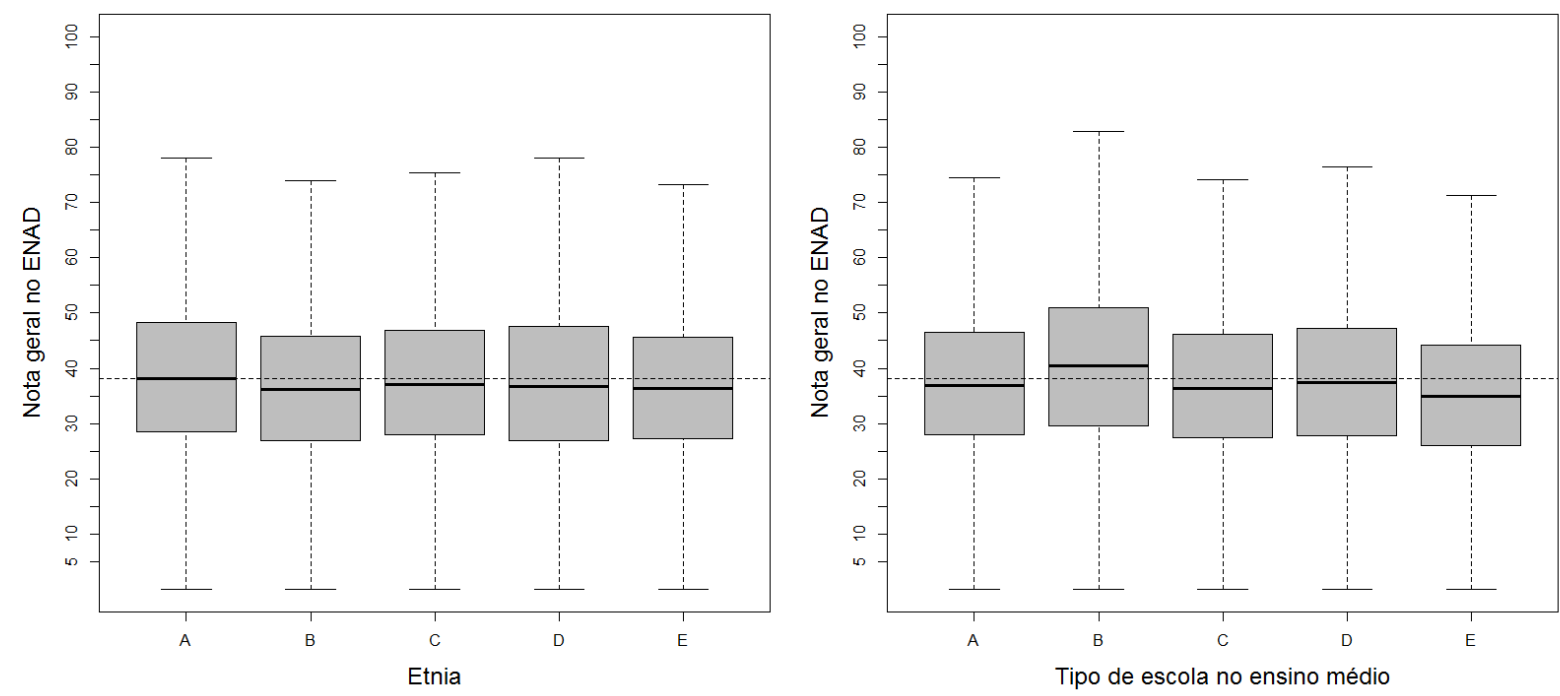

Fonte: Produção dos autores com base nos dados do ENADE.

Outras duas variáveis que possuem um importante papel para os objetivos deste trabalho, quais sejam, renda familiar dos respondentes e a escolaridade de seus pais, serão tratadas a seguir. A Tabela 2 contém os valores médios das notas no ENADE para cada subgrupo de alunos ao estratificá-los pelos níveis de Renda familiar e Escolaridade dos pais. As categorias de Renda familiar são: $A=$ Nenhuma; $B=$ Até 1,5 salário mínimo; $C=$ Acima de 1,5 até 3 salários mínimos; $\mathrm{D}=$ Acima de 3 até 4,5 salários mínimos; $\mathrm{E}=$ Acima de 4,5 até 6 salários mínimos; $F=$ Acima de 6 até 10 salários mínimos; $\mathrm{G}=$ Acima de 10 até 30 salários mínimos, e; Acima de 30 salários mínimos. Para Escolaridade dos pais, temos: A = Pai e mãe não possuem curso superior; $B=$ Somente o pai possui curso superior; $C=$ Somente a mãe possui curso superior e,; D = Pai e mãe possuem curso superior. Ressalta-se que tanto o pai como a mãe não possuem formação superior para quase $70 \%$ dos respondentes. Ilustra-se, também, a partir da Tabela 2, uma possível interação entre estas duas últimas variáveis no sentido de que o efeito que o aumento da renda exerce sobre o aumento da nota do ENADE depende da escolaridade dos pais. Isto é, para os pais com nenhuma escolaridade, a renda exerce impacto sobre a nota do ENADE. Já para os casos em que ambos os pais possuem o nível superior, o impacto da renda sobre a nota do ENADE é pouco expressivo. Para melhor visualizar isto, considere analisar separadamente as notas médias para o grupo de escolaridade dos pais na categoria A (pai e mãe sem formação superior). Para estes, observa-se um salto de aproximadamente 5 unidades na média do ENADE se passarmos da categoria 'nenhuma renda' para a categoria acima de 30 salários mínimos' (H). Porém, se a categoria de escolaridade dos 
Indicadores educacionais no Ensino Superior Brasileiro: possíveis articulações entre desempenho e características do alunado

pais for D (pai e mãe com formação superior), observamos um salto na média bem menos expressivo, inferior a 1 unidade. A Figura 2(A) pode auxiliar na percepção desta possível interação entre renda e escolaridade dos pais. Para tanto, foram inseridas linhas horizontais tracejadas para indicar as medianas mais elevadas dentro de cada categoria de escolaridade dos pais.

Tabela 2 - ENADE médio por categoria das variáveis Escolaridade dos pais e Renda familiar

\begin{tabular}{lccccc|c}
\hline \multicolumn{7}{c}{ Escolaridade dos Pais } \\
\cline { 2 - 7 } & \multicolumn{7}{c}{ A } & B & C & D & Global \\
\hline & A & 34,74 & 38,60 & 38,21 & 40,07 & 36,68 \\
Renda Familiar & B & 35,60 & 36,67 & 37,20 & 39,14 & 35,98 \\
& C & 36,33 & 37,95 & 37,09 & 38,73 & 36,59 \\
& D & 37,21 & 38,09 & 38,14 & 39,22 & 37,47 \\
& E & 37,73 & 39,07 & 38,98 & 39,99 & 38,17 \\
& F & 38,74 & 39,70 & 39,68 & 40,65 & 39,25 \\
& G & 40,20 & 41,04 & 40,76 & 42,01 & 41,01 \\
& H & 40,07 & 39,95 & 39,45 & 40,94 & 40,52 \\
\hline
\end{tabular}

Fonte: Instituto Nacional de Estudos e Pesquisas Educacionais Anísio Teixeira

Para os gráficos de caixa em amarelo (pai e mãe sem formação superior), observa-se que a linha tracejada dista cerca de 5 unidades da mediana do grupo de menor renda familiar. Agora, se consideradas as caixas do grupo em que ambos, pai e mãe, possuem curso superior, observarmos uma diferença bem menor entre os valores medianos do ENADE se comparados os grupos de renda mais alta com os de renda mais baixa. Outro achado, por sua vez curioso, é que as diferenças das medianas dos grupos de renda $\mathrm{C}$, $\mathrm{D}$ e $\mathrm{E}$, pelas dos grupos $\mathrm{G}$ e $\mathrm{H}$, são quase constantes ao longo das três últimas faixas de escolaridade. Ou seja, isto não se mantém quando fazemos tal comparação usando as médias ao invés das medianas. A Tabela 2 contém o ENADE médio para cada categoria de renda e escolaridade dos pais. Observamos que a interação entre estas duas variáveis é evidente para todos os níveis de renda. Outro ponto que merece ser ressaltado é que parece haver um efeito compensatório produzido por esta interação. Ou seja, quando ambos, pai e mãe, não possuem curso superior, porém sua família possui a mais elevada categoria de renda, então a nota média no ENADE foi de 40,07. Se fizermos a inversão, pais com escolaridade elevada e com renda familiar muito baixa, observa-se, igualmente, um ENADE médio de 40,07. No entanto, a combinação favorável de alta renda com alta escolaridade dos pais não eleva a nota média em mais do que uma unidade. Esse possível efeito compensatório será melhor discutido na Seção 4.4. 
Com respeito à variável Situação de trabalho, as categorias de resposta, seguidas pelas frequências relativas com que ocorreram, são: $\mathrm{A}=$ Não estou trabalhando $(24,37 \%)$; $\mathrm{B}=$ Trabalho eventualmente $(4,28 \%) ; \mathrm{C}=$ Trabalho até 20 horas semanais $(4,8 \%) ; \mathrm{D}=$ Trabalho mais de 20 horas semanais e menos de 40 horas semanais $(14,63 \%)$, e; $\mathrm{E}=$ Trabalho em tempo integral - 40 horas semanais ou mais $(51,92 \%)$. Como já discutido na Seção anterior, é de se esperar que alunos que trabalham venham a apresentar notas no ENADE inferiores se comparados com os que não trabalham. A Figura 2(B) evidencia que os que não trabalhavam, caixas amarelas, de fato apresentam notas medianas superiores em cerca de 3 unidades comparativamente aos que trabalham, chegando ao patamar de 43,2 para os de categoria de renda $\mathrm{G}$ ou $\mathrm{H}$. É interessante notar que, dentre os que trabalham, independentemente da quantidade de horas semanais exercidas, as notas medianas foram sempre próximas, o que é destacado com a linha horizontal pontilhada que passa pela nota 39,9. Também observa-se que as diferenças entre os que não trabalhavam e os que trabalhavam é amenizada quando a renda familiar é elevada, ou seja, também parece coerente que exista interação entre renda familiar e situação de trabalho.

Figura 2 - ENADE 2012: Gráfico (A) - por renda e escolaridade dos pais; Gráfico (B) - por renda e situação de trabalho

(A)

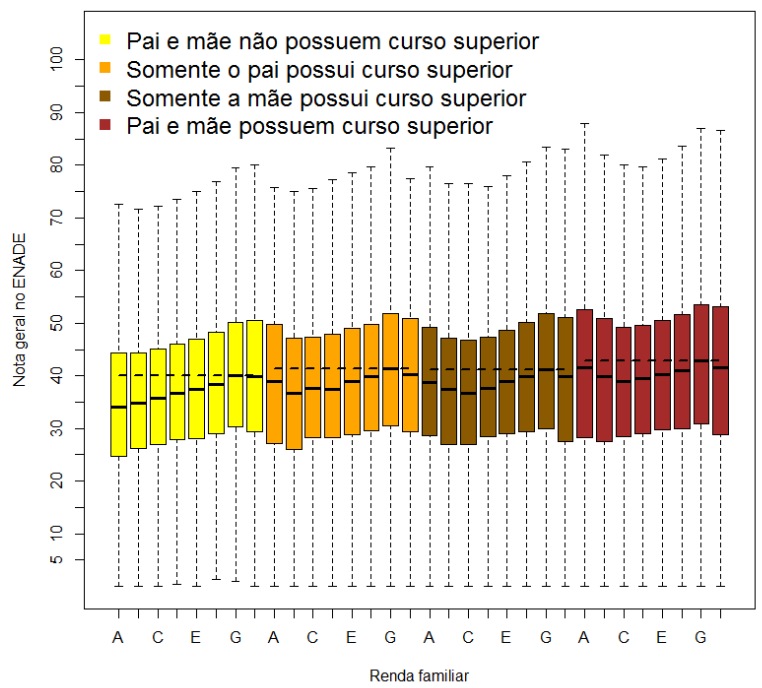

(B)

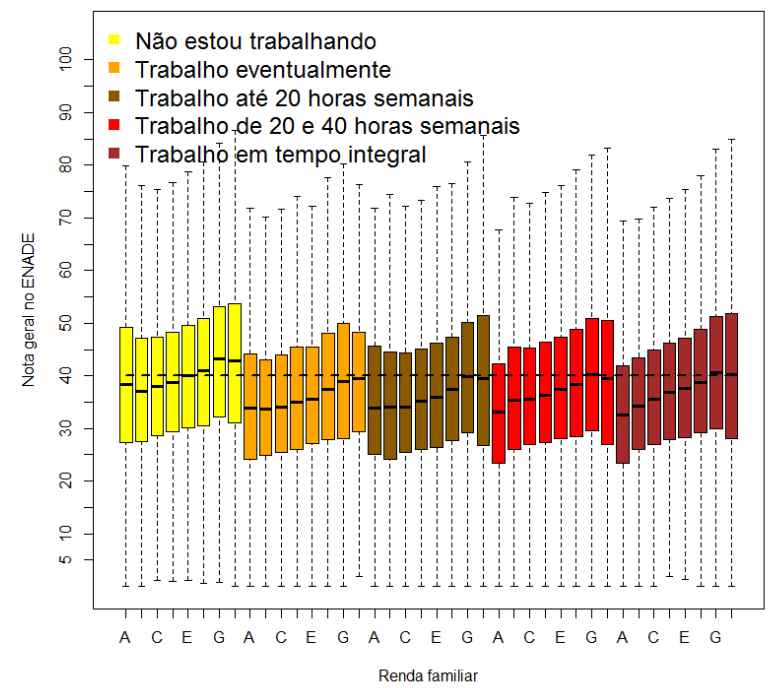

Fonte: Produção dos autores com base nos dados do ENADE. 
Indicadores educacionais no Ensino Superior Brasileiro: possíveis articulações entre desempenho e características do alunado

\subsection{Modelo linear proposto}

Feitas as devidas considerações sobre as evidências empíricas que suportam as relações previstas pela interpretação sociológica da educação de que considera a origem social como condição primordial para se pensar a trajetória escolar, passa-se agora para a estimação do modelo de regressão linear. A relação linear entre a nota geral no ENADE, do aluno \$i\$, e as covariáveis relacionadas ao mesmo, é expressa pelo modelo:

$$
\text { ENADE }_{i}=\beta_{0}+\sum_{j_{1}=1}^{4} \beta_{j_{1}} \text { Etnia }_{i, j_{1}}+\sum_{j_{2}=1}^{3} \beta_{j_{2}} \text { Pais }_{i, j_{2}}+\sum_{j_{3}=1}^{4} \beta_{j_{3}} \text { TipoEmedio }_{i, j_{3}}+
$$

$+\beta_{12} \operatorname{Trab}_{i}+\sum_{j_{4}=1}^{7} \operatorname{Renda}_{i, j_{4}}\left(\beta_{j_{4}+12}+\sum_{j_{5}=1}^{3} \beta_{j_{4} \cdot j_{5}+19} \operatorname{Pais}_{i, j_{5}}+\beta_{j_{40}+j_{4}} \operatorname{Trab}_{i}\right)+$

$+\beta_{48}$ CategIES $_{i}+e_{i}$,

onde:

- Etnia $_{i, 1}=1$ se a etnia do aluno $i$ for da categoria B, do contrário, Etnia ${ }_{i, 1}=0$. Etnia $_{i, 2}=1$ se a etnia for C, do contrário, Etnia $a_{i, 2}=0$. E assim sucessivamente até a etnia E. Observe que o modelo não possui um coeficiente para a etnia A, pois esta foi usada como a categoria de referência com a qual as demais são comparadas;

- Pais $\mathrm{i}_{\mathrm{i}, 1}=1$ se apenas o pai do aluno i possui ensino superior, do contrário, Pais $\mathrm{s}_{\mathrm{i}, 1}=0$. Pais $\mathrm{s}_{\mathrm{i}, 2}=$ 1 se apenas a mãe possui ensino superior, do contrário, Pais $\mathrm{i}_{\mathrm{i}, 2}=0$. Se pai e mãe possuem formação superior, então Pais ${ }_{i, 3}=1$, do contrário, Pais $_{i, 3}=0$;

- TipoEmedio ${ }_{i, 1}=1$ se o tipo de escola em que o aluno i cursou o ensino médio for da categoria $\mathrm{B}$, do contrário, TipoEmedio ${ }_{\mathrm{i}, 1}=0$. $\mathrm{E}$ assim sucessivamente até TipoEmedio ${ }_{\mathrm{i}, 4}$ para a categoria $\mathrm{D}$;

- Trab $_{\mathrm{i}}=1$ se o aluno i trabalha pelo menos eventualmente, e Trab $\mathrm{i}_{\mathrm{i}}=0$ caso contrário;

- Renda $_{\mathrm{i}, 1}=1$ se o aluno $\mathrm{i}$ for da categoria de renda $\mathrm{B}$, do contrário, Renda $\mathrm{i}_{1,1}=0 . \mathrm{E}$ assim sucessivamente até Renda $_{\mathrm{i}, 7}$ para a categoria $\mathrm{H}$;

- CategIES $S_{\mathrm{i}}=1$ se a categoria administrativa da IES do aluno i é pública, e CategIES $\mathrm{i}_{\mathrm{i}}=0$ caso contrário.

A segunda linha da expressão (2) explicita a interação do tipo multiplicativa da renda familiar com a escolaridade dos pais e com a situação de trabalho. Esta construção concorda com a noção de que o efeito de se mudar da renda mais baixa (A), para alguma das demais 
categorias, depende da categoria de escolaridade dos pais e da situação de trabalho do aluno. $\mathrm{O}$ coeficiente da variável Renda $a_{i, j}$ é $\left(\beta_{j+12}+\eta\right)$, onde $\eta$ é um valor que varia de acordo com o nível de formação dos pais e da situação de trabalho do aluno.

\subsection{Metodologia para inferência estatística}

Na Seção 4.1 foi ressaltado que as notas medianas por categoria de renda, e por escolaridade dos pais Figura (2), apresentavam um comportamento diferente daquele observado com as médias amostrais Tabela (2). Isto ocorre pelo fato da média amostral ser fortemente influenciada por valores extremos. A mediana, além de ser imune ao efeito de valores extremos, é uma estatística que favorece uma interpretação probabilística sobre os valores centrais da distribuição de notas. Assim, visando fornecer uma modelagem para a média e para a mediana separadamente, serão apresentados os resultados para dois modelos de regressão distintos, um para a modelagem da média e outro para modelagem da mediana. Para a regressão sobre a média, a estimação dos parâmetros se dá pelo uso do convencional método dos mínimos quadrados ordinários (MQO). Para a regressão sobre a mediana, aplicou-se a abordagem da regressão quantílica (RQ) que, por sua vez, é robusta ao efeito de valores extremos e/ou atípicos. Para detalhes sobre a teoria de inferência estatística e computacional envolvidas na modelagem RQ, pode-se citar Koenker e Basset (1982, 2001); Koenker e Xiao (2002). Todos os cálculos necessários para estimação dos modelos foram possíveis pelo uso do software de programação estatística R (TEAM, 2014) que pode ser baixado gratuitamente em `http://cran.r-project.org/'.

\subsection{Resultados}

A Tabela 3 fornece as estimativas dos parâmetros $\beta_{0}, \beta_{1}, \ldots, \beta_{48}$ do modelo (2) para as abordagens RQ e MQO. A primeira coluna da Tabela 3 contém os rótulos de cada covariável e da segunda à quarta colunas temos as estimativas para o modelo por RQ para a mediana. Da quinta à sétima colunas tem-se as informações para o modelo por MQO. As colunas com os rótulos $\widehat{\beta}$ e $\hat{b}$ contém os coeficientes e os coeficientes padronizados dos modelos, respectivamente. Apesar dos betas diferirem ligeiramente entre as abordagens MQO e RQ, algumas conclusões gerais coincidem. Como exemplo, há a etnia de referência, branco(a), apresentando a média e mediana ambas maiores que as das demais categorias. Porém, enquanto a menor mediana ocorre para a categoria D (amarelo), que apresenta um valor cerca de 1,3 unidades inferior à categoria $\mathrm{A}$, a menor média ocorre na categoria $\mathrm{E}$ (indígena ou de origem indígena), estimada como algo em torno de 1,4 unidades inferior à da etnia A. Tal achado 
corrobora com as evidências encontradas em pesquisas anteriores que apontam aqueles que se declaram brancos como tendo resultados superiores a pardos, negros e amarelos, respectivamente.

Quanto ao efeito do tipo de ensino médio cursado pelo respondente, as estimativas mostram que tanto a mediana quanto a média são maximizadas dentre aqueles que cursaram o segundo grau integralmente em escola privada. Verifica-se, novamente, a confirmação de resultados já encontrados em pesquisas anteriores de que o ensino médio particular tem se destacado nas avaliações realizadas. Vale ressaltar que este efeito da escola privada é mais expressivo na mediana, que é elevada em cerca de 1,2 unidades em relação à categoria de referência (ensino médio integralmente em escola pública). Esta última se destaca em relação às outras categorias, apresentando uma média de 1 a 2,5 unidades maior. Com respeito à categoria administrativa das IES, as públicas fornecem tanto mediana quanto média expressivamente maiores que as privadas. As estimativas para mediana e média das públicas mostram-se cerca de 3,8 e 2,8 unidades, respectivamente, superiores às das privadas. Novamente, com esse estudo foi possível confirmar estudos anteriores que evidenciaram a presença de alunos com melhor desempenho nas IES públicas. 
Tabela 3 - Modelos de Regressão Linear para ENADE 2012

\begin{tabular}{|c|c|c|c|c|c|c|}
\hline \multirow[b]{2}{*}{ Coeficientes } & \multicolumn{2}{|r|}{ RQ } & \multicolumn{4}{|c|}{ MQO } \\
\hline & $\widehat{\boldsymbol{\beta}}$ & $\widehat{b}$ & valor-p & $\widehat{\boldsymbol{\beta}}$ & $\widehat{b}$ & valor-p \\
\hline (Intercepto) & 35,70 & 93,51 & $<10^{-6}$ & 35,86 & 128,52 & $<10^{-6}$ \\
\hline EtiniaB & $-1,00$ & $-9,44$ & $<10^{-6}$ & $-1,04$ & $-12,41$ & $<10^{-6}$ \\
\hline EtiniaC & $-0,50$ & $-8,19$ & $<10^{-6}$ & $-0,45$ & $-9,53$ & $<10^{-6}$ \\
\hline EtiniaD & $-1,30$ & $-6,33$ & $<10^{-6}$ & $-1,00$ & $-6,37$ & $<10^{-6}$ \\
\hline EtiniaE & $-0,80$ & $-2,37$ & 0,01761 & $-1,38$ & 5,31 & $<10^{-6}$ \\
\hline RendaB & 0,60 & 1,43 & 0,15138 & 1,14 & 3,71 & 0.00207 \\
\hline RendaC & 1,60 & 4,01 & 0,00006 & 1,94 & 6,64 & $<10^{-6}$ \\
\hline RendaD & 2,40 & 5,89 & $<10^{-6}$ & 2,56 & 8,59 & $<10^{-6}$ \\
\hline RendaE & 3,20 & 7,77 & $<10^{-6}$ & 3,16 & 10,29 & $<10^{-6}$ \\
\hline RendaF & 3,90 & 9,32 & $<10^{-6}$ & 3,74 & 12,37 & $<10^{-6}$ \\
\hline RendaG & 5,90 & 13,87 & $<10^{-6}$ & 5,43 & 17,34 & $<10^{-6}$ \\
\hline RendaH & 5,70 & 8,90 & $<10^{-6}$ & 5,66 & 12,56 & $<10^{-6}$ \\
\hline Esc_pais2 & 3,20 & 3,80 & 0,00015 & 2,94 & 4,38 & 0.000018 \\
\hline Esc_pais3 & 3,10 & 6,35 & $<10^{-6}$ & 2,43 & 4,47 & $<10^{-6}$ \\
\hline Esc_pais4 & 4,80 & 7,61 & $<10^{-6}$ & 3,85 & 8,54 & $<10^{-6}$ \\
\hline Se_trabSim & $-3,50$ & $-8,53$ & $<10^{-6}$ & $-3,17$ & $-8,21$ & $<10^{-6}$ \\
\hline Tipo_EnsMedioB & 1,20 & 15,95 & $<10^{-6}$ & 0,77 & 13,69 & $<10^{-6}$ \\
\hline Tipo_EnsMedioc & $-1,00$ & $-8,67$ & $<10^{-6}$ & $-0,99$ & $-11,11$ & $<10^{-6}$ \\
\hline Tipo_EnsMedioD & $-0,80$ & $-5,75$ & $<10^{-6}$ & $-0,89$ & $-8,50$ & $<10^{-6}$ \\
\hline Tipo_EnsMedioE & $-2,40$ & $-16,45$ & $<10^{-6}$ & $-2,46$ & $-19,49$ & $<10^{-6}$ \\
\hline Categ_IESPublica & 3,80 & 37,78 & $<10^{-6}$ & 2,84 & 44,04 & $<10^{-6}$ \\
\hline RendaB:Esc_pais2 & $-2,00$ & $-2,11$ & 0,0347 & $-2,38$ & $-3,15$ & 0.001617 \\
\hline RendaC:Esc_pais2 & $-1,80$ & $-2,02$ & 0,04318 & $-1,76$ & $-2,51$ & 0.011947 \\
\hline RendaD:Esc_pais2 & $-3,00$ & $-3,44$ & 0,00058 & $-2,47$ & $-3,55$ & 0.000381 \\
\hline RendaE:Esc_pais2 & $-2,20$ & $-2,50$ & 0,01239 & $-2,11$ & $-3,01$ & 0.002619 \\
\hline RendaF:Esc_pais2 & $-2,60$ & $-2,96$ & 0,00312 & $-2,51$ & $-3,63$ & 0.000281 \\
\hline RendaG:Esc_pais2 & $-3,00$ & $-3,38$ & 0,00072 & $-2,81$ & $-4,03$ & 0.000049 \\
\hline RendaH:Esc_pais2 & $-3,40$ & $-2,97$ & 0,00294 & $-3,66$ & $-4,51$ & $<10^{-6}$ \\
\hline RendaB:Esc_pais3 & $-1,20$ & $-2,01$ & 0,04462 & $-1,33$ & $-2,19$ & 0.028661 \\
\hline RendaC:Esc_pais3 & $-2,80$ & $-5,32$ & $<10^{-6}$ & $-2,13$ & $-3,77$ & 0.000166 \\
\hline RendaD:Esc_pais3 & $-2,70$ & $-5,00$ & $<10^{-6}$ & $-1,98$ & $-3,51$ & 0.000453 \\
\hline RendaE:Esc_pais3 & $-2,30$ & $-4,30$ & 0,00002 & $-1,76$ & $-3,10$ & 0.001944 \\
\hline RendaF:Esc_pais3 & $-2,60$ & $-4,93$ & $<10^{-6}$ & $-2,07$ & $-3,69$ & 0.000225 \\
\hline RendaG:Esc_pais3 & $-3,20$ & $-5,87$ & $<10^{-6}$ & $-2,60$ & $-4,52$ & 0.000003 \\
\hline RendaH:Esc_pais3 & $-3,70$ & $-4,69$ & $<10^{-6}$ & $-3,68$ & $-5,00$ & $<10^{-6}$ \\
\hline RendaB:Esc_pais4 & $-1,40$ & $-1,84$ & 0,06511 & $-1,39$ & $-2,48$ & 0.013020 \\
\hline RendaC:Esc_pais4 & $-2,90$ & $-4,33$ & 0,00001 & $-2,38$ & $-4,82$ & $<10^{-6}$ \\
\hline RendaD:Esc_pais4 & $-3,10$ & $-4,48$ & 0,00001 & $-2,72$ & $-5,58$ & $<10^{-6}$ \\
\hline RendaE:Esc_pais4 & $-3,10$ & $-4,55$ & 0,00001 & $-2,56$ & $-5,27$ & $<10^{-6}$ \\
\hline RendaF:Esc_pais4 & $-3,60$ & $-5,44$ & $<10^{-6}$ & $-2,92$ & $-6,21$ & $<10^{-6}$ \\
\hline RendaG:Esc_pais4 & $-3,70$ & $-5,59$ & $<10^{-6}$ & $-3,26$ & $-6,91$ & $<10^{-6}$ \\
\hline RendaH:Esc_pais4 & $-4,40$ & $-5,45$ & $<10^{-6}$ & $-4,12$ & $-7,33$ & $<10^{-6}$ \\
\hline RendaB:Se_trabSim & 1,20 & 2,63 & 0,00861 & 1,10 & 2,63 & 0.008568 \\
\hline RendaC:Se_trabSim & 1,50 & 3,49 & 0,00049 & 1,40 & 3,50 & 0.000461 \\
\hline RendaD:Se_trabSim & 1,80 & 4,10 & 0,00004 & 1,80 & 4,47 & 0.0000088 \\
\hline RendaE:Se_trabSim & 1,60 & 3,61 & 0,0003 & 1,69 & 4,14 & 0.000043 \\
\hline RendaF:Se_trabSim & 2,00 & 4,49 & 0,00001 & 2,13 & 5,27 & $<10^{-6}$ \\
\hline RendaG:Se_trabSim & 1,40 & 3,12 & 0,00184 & 1,70 & 4,17 & 0.000012 \\
\hline RendaH:Se_trabSim & 0,60 & 1,04 & 0,3003 & 0,90 & 1,93 & 0.053087 \\
\hline
\end{tabular}

Fonte: Produção dos autores com base nos dados do ENADE.

Por fim, percebe-se que o efeito da renda familiar nas notas mediana e média do ENADE variam, consideravelmente, de acordo com as categorias de escolaridade dos pais. Tal efeito também interage com a situação de trabalho. Para analisar essas relações, separadamente, vamos primeiramente tratar da interação da renda com escolaridade dos pais, supondo a situação de trabalho fixa para um aluno que trabalhe. Os coeficientes estimados para todos as combinações do tipo 'Renda:Esc.pais' são todos negativos e, de modo geral, apresentam os 
Indicadores educacionais no Ensino Superior Brasileiro: possíveis articulações entre desempenho e características do alunado

menores valores (módulos maiores) para os casos em que pai e mãe possuem curso superior, dando-se a impressão de um efeito compensatório da alta escolaridade sobre a renda. Por exemplo, se pai e mãe não possuem ensino superior, então a mediana estimada para um aluno da categoria $\mathrm{H}$ de renda familiar será cerca de 5,7 unidades superior à mediana daqueles da categoria A de renda. Porém, se pai e mãe possuem formação superior, então a diferença estimada entre a mediana da categoria de renda $\mathrm{H}$ e mediana da categoria $\mathrm{A}$ será de $\$(5,7-$ $4,4+0,6)=1.9$. Este efeito compensatório, da escolaridade sobre a renda, praticamente anula (algumas vezes até reverte) as vantagens que as categorias de renda da $\mathrm{B}$ à $\mathrm{F}$ poderiam apresentar sobre as famílias que não possuem renda. Agora, para destacar o efeito da interação da renda familiar também com a situação de trabalho, suponha que o aluno não trabalhe. Com isso, a diferença de medianas entre as categorias H e A será de $(5,7-4,4)=1,3$, menos expressiva do que as 1,9 unidades iniciais. Em síntese, encontra-se que trabalhar trás um maior prejuízo sobre a nota para aqueles que possuem renda menor.

É preciso, igualmente, dar destaque ao fato do coeficiente da interação entre renda e trabalho não se mostrar significativo a $5 \%$ de confiança. $\mathrm{O}$ modelo RQ é robusto ao detectar que esta interação não se mostra significativa sequer para níveis de confiança extremamente altos, como de $30 \%$, por exemplo. Observe, também, que a tendência dos coeficientes das interações 'RendaX:TrabSim' se reverte de positiva para negativa após a faixa ' $\mathrm{X}=\mathrm{F}$ ' de renda, ou seja, o aumento da nota é desacelerado após a faixa de renda F. Outro achado que merece ser mencionado é a que a mediana daqueles com renda de até 1,5 salários mínimos não se difere significativamente dos que não possuem renda (valor-p=0,15138). Os coeficientes não significativos do modelo foram mantidos a fim de simplificar a interpretação dos termos de interação.

\section{Discussão}

As evidências encontradas ao longo desse estudo apontam para a confirmação da Teoria proposta por Bourdieu (2001) sobre a forte relação entre desempenho acadêmico e características socioeconômicas, na medida em que se identifica a presença da bagagem socialmente herdada e seus capitais cultural, social e econômico, atuantes de forma a criarem condições mais ou menos favoráveis diante da obtenção de boas notas. Isso pode ser visualizado, por exemplo, ao constatar-se que a renda, quando baixa, conjugada a uma situação do aluno estar trabalhando, produz o efeito de uma menor nota no ENADE. Ao contrário, alunos pertencentes a uma faixa de renda mais elevada, têm sua nota pouco afetada pelo fato de estarem 
trabalhando. Uma possível explicação hipotética para isso seria considerar que o trabalho nas classes mais elevadas teria características próprias, como aprendizado profissional, contribuição para os negócios da família, ao invés de autossustento. Nas classes mais elevadas, poderíamos supor a existência de regras mais flexíveis e ambiente profícuo para o aprendizado acadêmico, o que pode não acontecer nos casos de trabalho por motivo de autossustento ou sustento familiar. A propósito, em estudo recente, Nogueira (2004) investigou a trajetória escolar de famílias de empresários com alto capital econômico e identificou que muitos de seus filhos trabalhavam nas condições descritas acima. Encontrou-se, também, que o efeito da renda familiar nas notas mediana e média do ENADE variam, consideravelmente, de acordo com as categorias de escolaridade dos pais. Ou seja, quanto maior a escolaridade dos pais, melhores as notas no ENADE. Isso, também, encontra respaldo nos estudos feitos a partir de Bourdieu (2001), na medida em que considera o capital cultural como o elemento na bagagem familiar que teria maior impacto na definição do destino escolar. Nogueira e Nogueira (2002) corrobora com essa afirmação, o fato de encontrarmos que a renda pouco afeta a nota do ENADE se ambos os pais possuem ensino superior. No entanto, identificou-se, empiricamente, que um baixo capital cultural institucional (pais sem ensino superior completo) pode ser compensado por um alto capital econômico. Isso poderia ser justificado, talvez, pelo fato de o capital econômico permitir o acesso a bens e serviços que auxiliariam o aluno na acumulação de uma bagagem cultural, necessária a uma melhor perspectiva de trajetória acadêmica. Quanto aos demais fatores, evidenciou-se que ainda permanecem no ensino superior um número maior de alunos declarados brancos e que alcançam, concomitantemente, a maior nota no ENADE. Esse achado confirma estudos anteriores nos quais os alunos que se declaram brancos têm desempenho superior aos demais que se consideram pertencentes a outras etnias. Silva, Vendramini e Lopes (2010); Soares e Marotta (2009); Barros et al. (2001); Henriques (2001); Andrade e Dachs (2007); Carvalho e Waltenbeg (2014). Uma possível explicação para esse fato pode estar associado a estratificação social brasileira refletida no desigual acesso ao ensino superior, que vem sendo problematizado com a implantação do sistema de cotas como medida para que estudantes negros, indígenas e oriundos do sistema público de ensino passem a participar de um mecanismo de promoção de grupos e populações sub-representadas nas instituições de ensino superior. Carvalho e Waltenbeg (2014). Mas, mesmo após a implantação do sistema de cotas, o percentual de alunos brancos ainda é maior no ensino superior. Nas palavras de Oliveira e Melo-Silva (2010), em termos étnico-raciais, o perfil da população que atingiu a universidade é assim distribuído: $79 \%$ se autodenominam de cor branca; $16,8 \%$, parda e $2,4 \%$, negra. Outro dado evidenciado trata-se da nota também ser impactada positivamente 
Indicadores educacionais no Ensino Superior Brasileiro: possíveis articulações entre desempenho e características do alunado

pela situação de se ter estudado o ensino médio todo em escola privada. Ao passo que as notas são, também, maiores para alunos que cursam a graduação em IES públicas. Esses dois achados podem estar associados, hipoteticamente, ao fato de uma grande seletividade social no processo de ingresso nas IES, de forma a conduzir a um padrão de que os alunos mais preparados entrariam para o ensino superior público, reconhecidamente como mais disputado, com uma bagagem cultural maior, que os favoreceria do ponto de vista de perpetuar o sucesso acadêmico nos anos seguintes. Obviamente, que se trata de uma problematização inicial e que seriam necessários outros estudos para contribuir com essa discussão sobre o impacto dos anos estudados em escolas privadas sobre o bom desempenho no ensino superior.

A propósito, apesar de ficar claro o peso da origem social sobre o desempenho de estudantes do ensino superior, esse estudo abre portas para que outras investigações sejam realizadas, seja por seu caráter unicamente quantitativo e que poderia ser complementado por análises qualitativas e de caráter microscópico, seja por tratar de um tema complexo e multicausal. Assim, sem desconsiderarem-se os condicionantes socioeconômicos, poderiam ser investigadas, a partir desse estudo, causas outras atribuídas ao desempenho, como ação individual, estratégias familiares, constituição da trajetória escolar, entre tantos outros que engrandeceriam o debate sobre a trajetória acadêmica de alunos no ensino superior.

\section{Referências}

ALVES, Kelly Ludkiewicz. Desempenho escolar e práticas culturais familiares: a relação de alunos do ensino fundamental II com a disciplina de história. Revista Educação em Questão, Natal, v. 43, n. 29, p. 11-24, 2012.

ALVES, Maria Tereza Gonzaga. Dimensões do efeito das escolas: explorando as interações entre famílias e estabelecimentos de ensino. Est. Aval. Educ., São Paulo, v. 21, n. 46, p. 271296, 2010.

ALVES, Maria Tereza Gonzaga; SOARES, José Francisco. Contexto escolar e indicadores educacionais: condições desiguais para a efetivação de uma política de avaliação educacional. Educação e Pesquisa, Campinas, v. 39, n. 1, p. 177-194, jan./mar. 2001.

ANDRADE, Cibele Yahn de; DACHS, J. Norberto W. Acesso à educação por faixas etárias segundo renda e raça. Cadernos de Pesquisa, São Paulo, v. 37, n. 131, maio/ago. p. 399-422, 2007.

BARROS, Ricardo Paes de; MENDONÇA, Rosane; SANTOS, Daniel Domingues dos; QUINTAES, Giovani. Determinantes do desempenho educacional no Brasil., Rio de Janeiro: IPEA, 2001. (Texto para discussão, 834).

BONAMINO, Alice; ALVES, Fátima; FRANCO, Creso; CAZELLI, Sibele. Os efeitos das diferentes formas de capital no desempenho escolar: um estudo à luz de Bourdieu e de 
Coleman. Revista Brasileira de Educação, Rio de Janeiro, v. 15, n. 45, p. 487-594, set./dez. 2010.

BOURDIEU, Pierre. Os Três estados do capital cultural. In: NOGUEIRA, Maria Alice; CATANI, Afrânio (Org.). Escritos de educação. Petrópolis: Vozes, 2001. p. 71-79.

CARDOSO, Rute; SAMPAIO, Helena. Estudantes universitários e o trabalho. Revista Brasileira de Ciências Sociais, São Paulo, v. 9, 1994.

CARVALHO, Márcia. Marques de; WALTENBERG, Fábio Domingues. Desigualdade de oportunidades no acesso ao ensino superior brasileiro. 2014. Disponível em: <http://www.ufjf.br/encontroeconomiaaplicada/files/2014/05/DESIGUALDADEDEOPORTUNIDADES-NO-ACESSO-AO-ENSINO-SUPERIOR-NO.pdf.> Acesso em: 31 jun. 2014.

CASTRO, Maria Helena Guimarães; TIEZZI, Sérgio. A reforma do ensino médio e a implantação do Enem no Brasil. In: SCHWARTZMAN, Simon. Os desafios da educação no Brasil. Rio de Janeiro: Nova Fronteira, 2005.

CUNHA, Maria Amália de Almeida. Sociologia da educação. Belo Horizonte: Editora UFMG, 2010.

HENRIQUES, Ricardo. Desigualdade racial no Brasil: evolução das condições de vida na década de 90. Rio de Janeiro: IPEA, 2001. (Texto para discussão, 807).

INEP - Instituto Nacional de Pesquisa em Educação Anísio Teixeira. Censos do ensino superior. Brasília, 2008. Disponível em: <http://www.inep.gov.br>. Acesso em: 28 jul. 2014.

KOENKER, Roger; BASSETT, Gilbert. Robust tests for heteroscedasticity based on regression quantiles. Econometrica, Cleveland, v. 50, p. 43-61, 1982.

KOENKER, Roger; BASSETT, Gilbert. Regression quantiles. Econometrica, Cleveland, v. 46, p. 33-50, 2001.

KOENKER, Roger, XIAO, Zheng. Inference on the quantile regression process".

Econometrica, Cleveland, v. 70, p. 1583-1612, 2002.

LAROS, Jacob. A.; MARCIANO, João Luiz Pereira; ANDRADE, Josemberg Moura de. Fatores que afetam o desempenho na prova de matemática do Saeb: um estudo multinível. Avaliação Psicológica, Porto Alegre, v. 9. n. 2, p. 173-186, 2010.

LORDÊLO, José Albertino Carvalho. Perfil, desempenho escolar, exclusão e inclusão no curso de administração da UFBA: locus para ação afirmativa? Revista Diálogos Possíveis, Salvador, v. 2, p. 199-217, 2004.

MORAES, Regina Aparecida; NUNES, Silma do Carmo. Uma reflexão para os supervisores escolares sobre o desempenho de alunos de escolas particulares e públicas no processo seletivo da UFU 2009/1. Revista da Católica, Uberlândia, v. 2, n. 3, p. 412-425, 2010.

NOGUEIRA, Maria Alice. Favorecimento econômico e excelência escolar: um mito em questão. Revista Brasileira de Educação, Rio de Janeiro, n. 26, p. 133-144, maio/ago. 2004.

NOGUEIRA; Cláudio Marques Martins; NOGUEIRA, Maria Alice. A sociologia da educação de Pierre Bourdieu: limites e contribuições. Educação e Sociedade, Campinas, v. 23, n. 78, p. 15-36, abr. 2002.

NOGUEIRA, Maria Alice; NOGUEIRA; Cláudio Marques Martins. Bourdieu e a educação. Belo Horizonte: Autêntica, 2009. 
OLIVEIRA, Melina del Arco; MELO-SILVA, Lucy Leal. Estudantes universitários: a influência das variáveis socioeconômicas e culturais na carreira. Psicol. Esc. Educ., São Paulo, v. 14, n. 1, pp. 23-34, jan./jun. 2010.

ORTEGA, Eliane Maria Vani. O ensino médio público e o acesso ao ensino superior. Estudos em avaliação educacional, São Paulo, n. 23, p. 153-176, jan./jun. 2001.

SILVA, Marjorie Cristina Rocha da; VENDRAMINI, Claudette Maria Medeiros; LOPES, Fernanda Luiza. Diferenças entre gênero e perfil socio-econômico no exame nacional de desempenho do estudante. Avaliação, Campinas; Sorocaba, v. 15, n. 3, p. 185-202, 2010.

SOARES, José Francisco; MAROTTA, Luana. Desigualdades no sistema de ensino fundamental brasileiro. In: HENRIQUES, Ricardo. et al. (Orgs.). Educação básica no Brasil: construindo o país do futuro. Rio de Janeiro: Elsevier, 2009. p. 73-91.

SOARES, José Francisco; MARTINS, Maria Inês; ASSUNÇÃO, Cibele Noronha Behrens. Heterogeneidade acadêmica dos alunos admitidos na ufmg e puc-mg. Estudos em Avaliação Educacional, São Paulo, n. 17, p. 61-72, 1998.

TEAM R CORE. R. A language and environment for statistical computing. $R$ Foundation for Statistical Computing. Vienna, Austria, 2014. Disponível em: <http://www.Rproject.org.> ISBN 3-900051-07-0. Acesso em: 31 jun. 2014.

Anandra Santos Ribeiro de Oliveira Universidade Federal de Ouro Preto | Instituto de Ciências Humanas e Sociais | Departamento de Educação Mariana | MG | Brasil. Contato: anandraribeiro@gmail.com

ORCIID ID 0000-0003-0856-9368

Ivair Ramos Silva Universidade Federal de Ouro Preto| Instituto de Ciências Exatas e Biológicas| Departamento de Estatística Ouro Preto | MG | Brasil. Contato: ivairest@gmail.com ORCID (D) 0000-0003-2701-8924 